\title{
Implementation Strategy of MPEG-2 Audio Decoder and Efficient Multichannel Architecture
}

\author{
Tsung-Han Tsai, Liang-Gee Chen, Ruei-Xi Chen \\ Department of Electrical Engineering, \\ National Taiwan University, Taipei, Taiwan, R.O.C.
}

\begin{abstract}
The paper describes an MPEG-2 audio decoder applied in multichannel extension. In the analysis of intelligent implementation strategy, the decoder can be divided into two hardwareoriented architecture stages. Also, an efficient architecture for multichannel processor is presented. The decoder is developed for the approach for simplicity and low-cost design.
\end{abstract}

\section{Introduction}

Digital audio coding has become an important technique in recent audio industry. One of these audio coding techniques, the ISO MPEG-2 audio standard, has developed a world-wide standard audio coding algorithm which can significantly reduce the requirements of transmission bandwidth and data storage with low distortion. In this paper, we present a novel MPEG-2 audio decoder which is capable of decoding MPEG-2 standard multichannel audio bit stream with an efficient architecture. According to the approach for simplicity and low-cost design, we take use of the dedicated hardware approach (ASIC), which provides a more efficient VLSI solution than commercial programmable design do. The decoder is developed for multichannel extension applications, without any external memory support.

\section{MPEG-2 Multichannel Decoding Process}

The MPEG-2 audio coding standard is an extension of MPEG-1 [1], [2]. Emphasis of the new activity is on multichannel and multilingual audio and on an extension of the existing standard to lower sampling frequencies and lower bit rates. Besides, backward compatibility is the key aspects to ensure the existing two channel decoders will still be able to decode compatible stereo information from five multichannel signals. This implies the provision of compatibility matrices, using adequate inverse matrix coefficients. Figure 1 shows how the operation of MPEG-2 audio codec and the coding relationships 


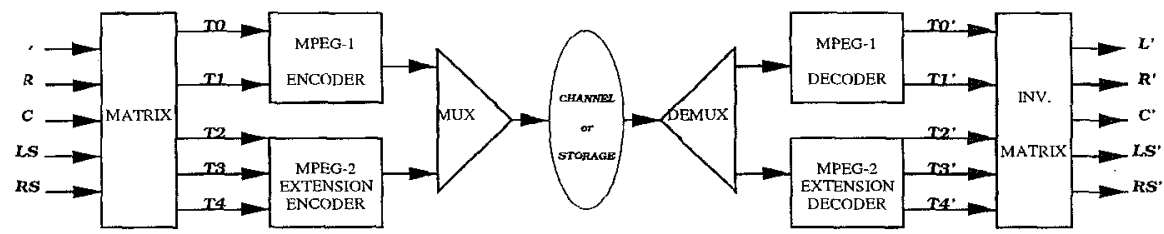

MPEG-2 ENCODER

MPEG-2 DECODER

Figure 1: Backward Compatible for MPEG-2 Audio Codec

among the five audio channels (L, R, C, LS, RS), the two basic channels (T0, T1) and the three extended channels (T2, T3, T4).

A matrixing operation of five channels in encoder yields two compatible stereo signals, L0, and R0. The signals L0 and R0 are transmitted in MPEG1 format in transmission channels $\mathrm{T} 0$ and $\mathrm{T} 1$. Channels $\mathrm{T} 2, \mathrm{~T} 3$, and T4 together form the multichannel extension signal. It is realised by exploiting the ancillary data field of the MPEG-2 data structure for the provision of the multichannel extended channels, as described in Figure 2.

\section{Decoder Implementation Strategy}

The basic block diagram for MPEG-2 audio decoder is shown in Figure 3 . First, the deformating process extracts the coded samples, multichannel processing modes and other control information. These samples are interpreted into quantized samples in inverse quantization. Then, multichannel processor reconstructs subband samples with quantized samples and other multichannel related information. Last, a set of 32 scaled-up subband samples are fed into the subband synthesis filter to reconstruct the five channel output PCM audio signals.

\subsection{Computation Analysis}

MPEG-2 audio decoding algorithm contains highly overheads of computation and complex control. Referring to the computation, Table 1 illustrates the most common arithmetic operations in MPEG-2 audio decoding. Each decoding classification block can be further divided into some functions. In inverse quantization (IQ), coded samples can be reconstructed into the multichannel samples by requantization and rescalizaiton functions. In multichannel processing (MC), the subband samples can be reconstructed by four main functions, such as dynamic crosstalk, dynamic transmission channel switching, dematrix and denormalization. The MC processing is described in Figure 4. At last stage, synthesis subband filter takes the inverse transform in the 


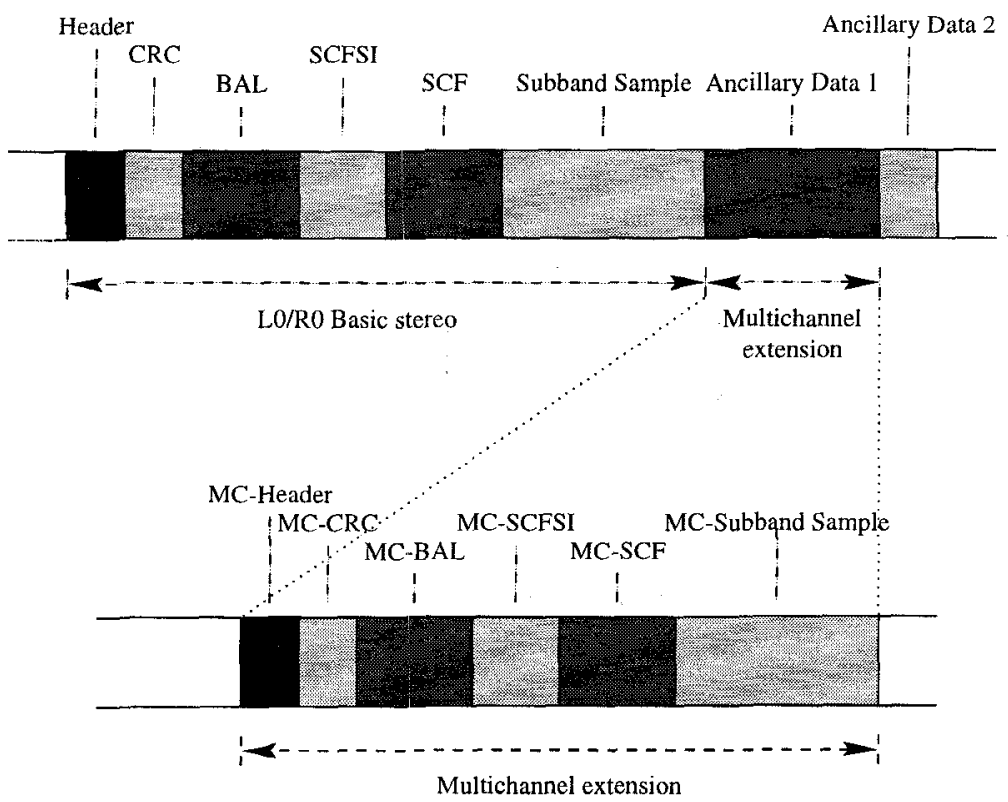

Figure 2: Data Structure of MPEG-2 Multichannel Bit Stream

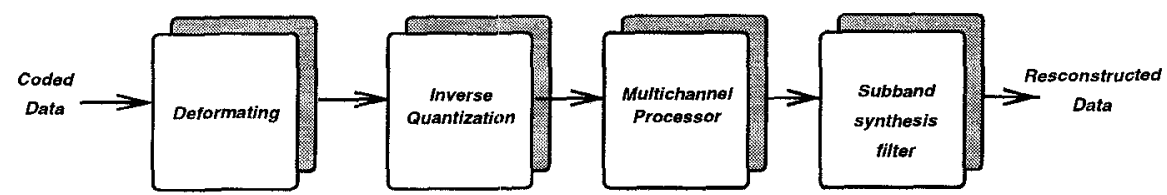

Figure 3: Block Diagram of the Decoder

Table 1: Arithmetic Operations in MPEG-2 Decoding

\begin{tabular}{|c||c||c|}
\hline Classification & Function & Required Arithmetic Operations \\
\hline \hline IQ & Requantization & $y=(x+a) b$ \\
& Rescalization & $y=a x$ \\
\hline MC & Dynamic Crosstalk & $y_{i}=s_{i} x_{j}$ \\
& DTCS & $y_{i}=x_{j}$ \\
& Dematrixing & $y=x_{i}-x_{j}-x_{k}$ \\
& Denormalization & $y=a x$ \\
\hline Syn. Subband & IMDCT & $y=a x+b, y=\sum_{i} c_{i} x_{i}$ \\
& IPQMF & $y=a x, y=\sum_{i} w_{i}$ \\
\hline
\end{tabular}




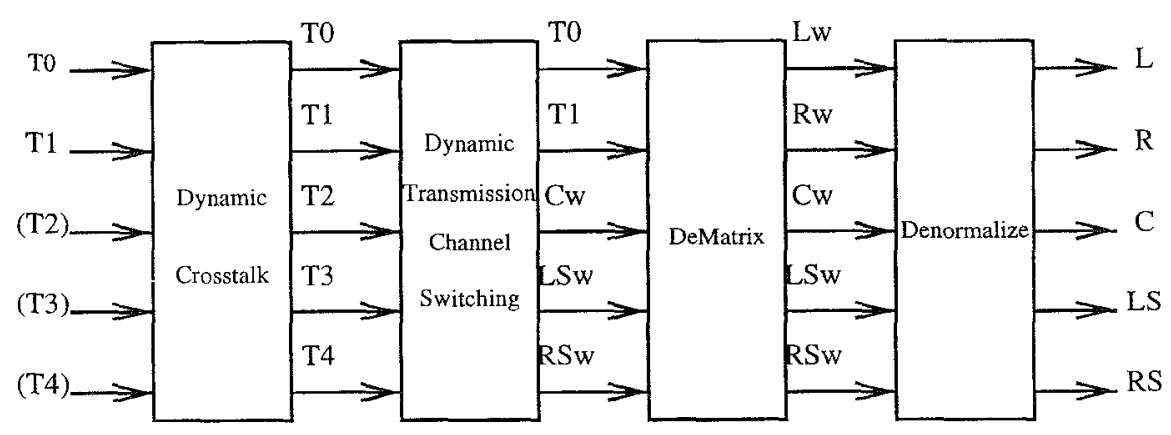

Figure 4: Multichannel Decoding Processing

Table 2: Distinctions Between Pre-processing and Post-processing stage

\begin{tabular}{|c||c||c|c|}
\hline Stage & Computation & Control & Memory Requirement \\
\hline \hline Pre-processing & few & complex & few \\
(IQ , MC) & $(7 \%)$ & & \\
\hline Post-processing & large & simple & large \\
(Synthesis Subband) & $(93 \%)$ & & \\
\hline
\end{tabular}

inverse Modified Discrete Cosine Transform (IMDCT), and implements the polyphase synthesis subband operations in the inverse Pseudo Quadrature Mirror Filter (IPQMF).

\subsection{Decomposition of Decoding Processing}

A similar analysis of the arithmetic operations in decoding algorithm shows that multiplication and addition are the most common operations. Such as the IMDCT module, a $32 \times 64$ transform can be realized by the multiplieraccumulate computation(MAC) directly. Also, a window operation with 512tap FIR is realized in IPQMF as the MAC operations. If a operation is defined as data access, store, add, shift, or multiply in most DSP applications, these two modules of synthesis subband occupy about $93 \%$ computation power of the whole decoding process.

Although most of the computation amount in whole decoding process are focused on synthesis subband filter, theses computation takes the advantages of simple control and regular data flow. Besides, the inverse quantization (IQ) and multichannel processing (MC) only occupy $7 \%$ computation amount of whole decoding process, but induce complex control and irregular data flow.

In order to improve the system performance, we develop an efficient implementation strategy. The decoding block diagram, as shown in Figure 3, 


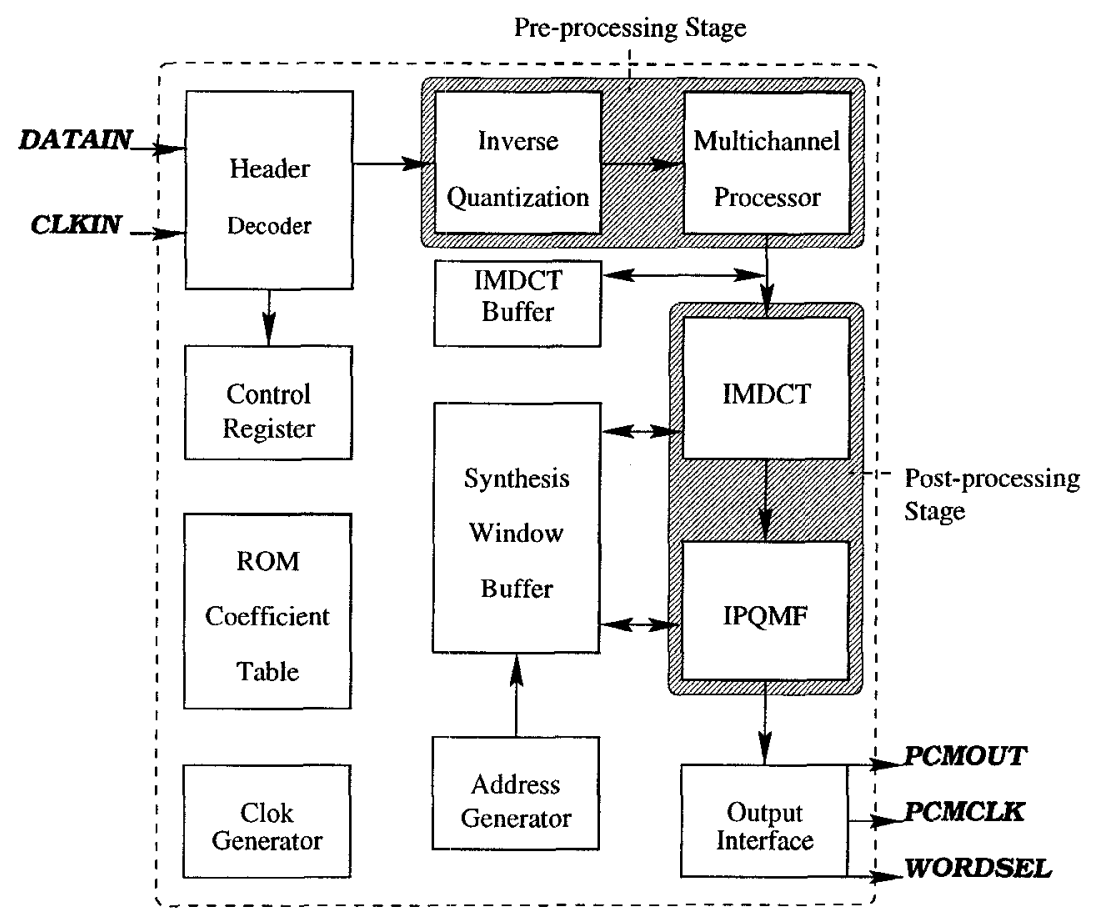

Figure 5: Overall Architecture Diagram

may be divided into two distinct stage, referred to here as pre-processing and post-processing stage. Pre-processing stage mainly includes the IQ and MC processing. Post-processing stage refers to the synthesis subband filter. The distinctions between these two stages are illustrated in Table 2 .

For pre-processing stage, it uses few computation power of whole decoding process and a relatively small amount of memory. But complex control will be induced, especially in MC decoding processor.

In contrast with the pre-processing stage, post-processing stage uses large computation power of whole decoding process with a simple and regular control, and a large amount of memory for synthesis window buffer. Thus, the pre-processing stage, especially for the multichannel processor, may be implemented by an efficient architecture with smooth data arrangement and efficient flow control. The post-processing stage may be implemented by high performance data path with fully hardware utilization. The architecture design issues for two processing stages will be introduced in next section. 


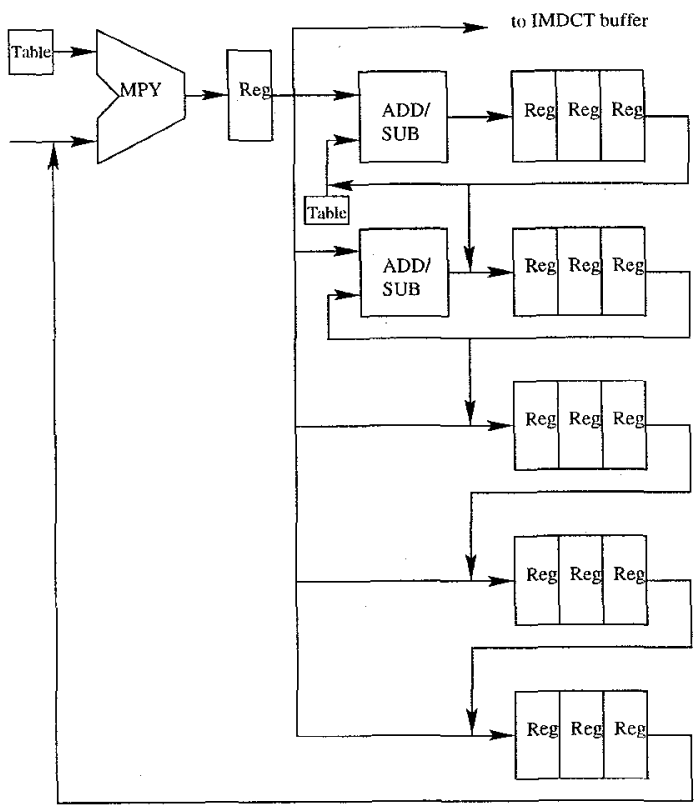

Figure 6: Efficient Architecture for Pre-processing Stage

\section{Architecture Design}

According to the approaches for simplicity and low-cost design, we take use of the dedicated hardware approach (ASIC), which provides a more efficient VLSI solution than commercial programmable design do.

Figure 5 describes the overall architecture diagram of our proposed design. It includes 5 primary modules called: header decoder, inverse quantization, multichannel processor, IMDCT module and IPQMF module. The header decoder interprets header information from audio bit-stream (DATAIN), then extracts multichannel processing modes and some control informations to store in control register. The pre-processing stage performs the inverse quantization and multichannel processing operations to reconstruct the subband samples. These samples are stored in IMDCT buffer. The IMDCT buffer, a ping-pong mode buffer, can be viewed as a output buffer of the pre-processing stage and the input buffer of the post-processing stage. A synthesis window buffer is supported for the implementation of synthesis subband functions in post-processing stage.

\subsection{Architecture Design for Pre-processing Stage}

Pre-processing stage can be implemented by the efficient architecture as shown in Figure 6. It mainly solves problems of complex control and irregu- 
Table 3: Decoder Memory Summary

\begin{tabular}{|c||c|}
\hline RAM Data Structure & Words \\
\hline \hline IMDCT Buffer & 128 \\
\hline Synthesis Window Buffer & 2560 \\
\hline
\end{tabular}

\begin{tabular}{|c||c|}
\hline ROM Data Structure & Words \\
\hline \hline Transform tables & 384 \\
\hline Other lookup tables & 256 \\
\hline
\end{tabular}

lar data flow for multichannel processing. Three registers per one channel is supported for Layer-2 decoding applications [1]. Only one multiplier and two adder/subtractor is used as a two-stage pipelined structure. Some quantized, rescalized, dematrixing and denormalization coefficients are stored in tables. This architecture takes the advantages of the efficient control strategy, and the smooth data flow without frequently memory access.

\subsection{Efficient Considerations for Post-processing Stage}

The synthesis subband filter operation take a large overheads of computation in whole decoding process. Thus it is necessary to reduce the computation amount by some fast algorithm [3]. We adopt the modified fast IMDCT algorithm to reduce the multiplier-accumulate computation into $1 / 4$ amount of the ISO suggestion method. Moreover, the required size for the synthesis window buffer in which the QMEF data V stored can be reduced only to 512 words per channel, instead of the original size of 1024 words per channel. The decoder memory is summarized in Table 3.

The IMDCT and IPQMF modules, divided from synthesis subband filter, mainly contain an identical multiplier-accumulator (MAC) respectively. The architecture of post-processing is more simple and regular than pre-processing stage. Also, by taking into account efficiency in hardware utilization and high performance, two identical MAC units perform a two-stage pipelined of structure as illustrated in Figure 7. With two identical pipeline stage of 512 clock cycles, $100 \%$ hardware utilization in post-processing can be feasible. This architecture is based on the goal for simplicity and low-cost design, without any external memory support. 


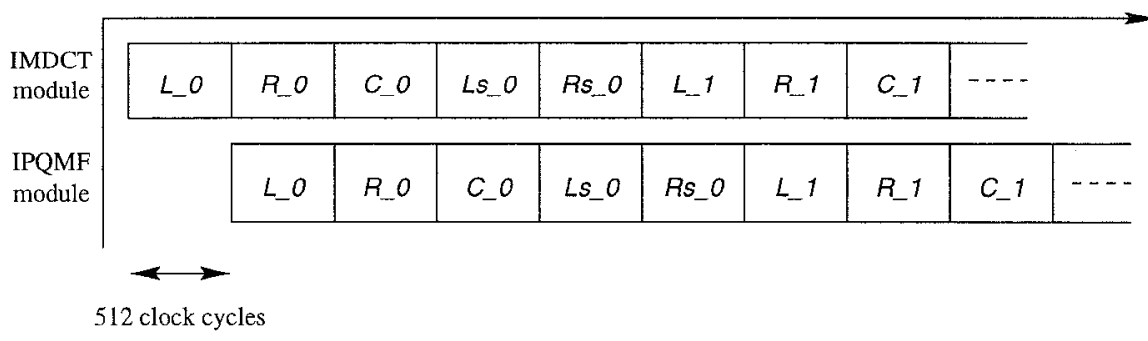

Figure 7: Two Stage Pipelined Reservation Table for Synthesis Subband Filter

\section{Conclusions}

We have designed an MPEG-2 audio decoder. Based on our design strategy, we divided the decoder into pre and post-processing stages. According to the detail discussions and hardware considerations, these two stages can be implemented in distinct approaches. Besides, an efficient multichannel processor architecture for pre-processing stage is proposed. The decoder is developed for the goals of simplicity and low-cost design.

\section{References}

[1] MPEG, "ISO CD 11172-3: coding of moving pictures and associated audio for digital storage media at up to about $1.5 \mathrm{Mb} / \mathrm{s}$ ", Nov 1991.

[2] MPEG, "ISO CD 13818-3: coding of moving pictures and associated audio for digital storage media at up to about $1.5 \mathrm{Mb} / \mathrm{s}$ ", Nov 1994.

[3] T. H. Tsai, T. H. and Chen, and L. G. Chen, "An MPEG audio decoder chip," IEEE Transactions on Consumer Electronics., vol. 41, no. 1, pp. 89-96, Feb 1995. 